\title{
UNIFORM BOUNDS AND SYMBOLIC POWERS ON SMOOTH VARIETIES
}

\author{
LAWRENCE EIN, ROBERT LAZARSFELD, AND KAREN E. SMITH
}

\section{INTRODUCTION}

The purpose of this note is to show how one can use multiplier ideals to establish effective uniform bounds on the multiplicative behavior of certain families of ideal sheaves on a smooth algebraic variety. In particular, we prove a quick but rather surprising result concerning the symbolic powers of radical ideals on such a variety.

Let $X$ be a non-singular quasi-projective variety defined over the complex numbers $\mathrm{C}$, and let $Z \subseteq X$ be a reduced subscheme of $X$. Denote by

$$
\mathfrak{q}=\mathcal{I}_{Z} \subseteq \mathcal{O}_{X}
$$

the ideal sheaf of $Z$, so that $\mathfrak{q}$ is a sheaf of radical ideals. We shall be concerned with the symbolic powers $\mathfrak{q}^{(m)}$ of $\mathfrak{q}$. According to a well-known theorem of Zariski and Nagata (see [5], Chapter 3.9) $\mathfrak{q}^{(m)}$ can be described as the sheaf of all function germs vanishing to order $\geq m$ at a general point of each irreducible component of $Z$ (or equivalently at every point of $Z$ ):

$$
\mathfrak{q}^{(m)}=\left\{f \in \mathcal{O}_{X} \mid \operatorname{ord}_{x}(f) \geq m \text { for all } x \in Z\right\} .
$$

It is evident that $\mathfrak{q}^{m} \subseteq \mathfrak{q}^{(m)}$, but in general of course the inclusion is strict. However Swanson [15] established (in a much less restrictive setting]) that there exists an integer $k=k(Z)$ depending on $Z$ such that

$$
\mathfrak{q}^{(k m)} \subseteq \mathfrak{q}^{m} \text { for all } m \in \mathbf{N}
$$

On geometric grounds this already seems rather striking since membership in the symbolic power on the left is tested at general smooth points of $Z$, whereas the actual power on the right reflects also its singular points. So one's first guess might be that the worse the singularities of $Z$, the larger one will have to take the coefficient $k(Z)$ to be. Surprisingly enough this is not the case, and in fact one has a uniform statement depending only on the codimension of $Z$ :

\footnotetext{
Research of the first author partially supported by NSF Grant DMS 99-70295.

Research of the second author partially supported by NSF Grant DMS 97-13149.

Research of the third author partially supported by NSF Grant DMS 96-25308.

${ }^{1}$ All of our results are local in nature, so there is no loss in taking $X$ to be an affine variety. In this case one can work with the coordinate $\operatorname{ring} \mathbf{C}[X]$ of $X$ in place of its structure sheaf $\mathcal{O}_{X}$.

${ }^{2}$ Swanson's theorem holds in particular on any normal variety over a field of any characteristic.
} 
Theorem A. Assume that every component of $Z$ has codimension $\leq e$ in $X$. Then

$$
\mathfrak{q}^{(m e)} \subseteq \mathfrak{q}^{m} \quad \text { for all } m \in \mathbf{N}
$$

In particular, if $\operatorname{dim} X=n$ then $\mathfrak{q}^{(m n)} \subseteq \mathfrak{q}^{m}$ for every radical ideal $\mathfrak{q} \subseteq \mathcal{O}_{X}$ and every natural number $m \geq 1$. One can see the Theorem as providing further confirmation of Huneke's philosophy [8] that there are unexpected uniform bounds lurking in commutative algebra.

Theorem A is a very simple application of the theory of multiplier ideals. In commutative algebra these were introduced by Lipman [13] in connection with the BriançonSkoda theorem. More general constructions, which we use here, have in the meantime become extremely important in the study of higher dimensional algebraic varieties (cf. [1], [2], [国, [14], [10], [12]). In brief, we consider families $\mathfrak{a}_{\bullet}=\left\{\mathfrak{a}_{k}\right\}$ of ideals $\mathfrak{a}_{k} \subseteq \mathcal{O}_{X}$ such as the symbolic powers $\mathfrak{q}^{(\bullet)}=\left\{\mathfrak{q}^{(k)}\right\}$ — satisfying the relations

$$
\mathfrak{a}_{\ell} \cdot \mathfrak{a}_{m} \subseteq \mathfrak{a}_{\ell+m} \text { for all } \ell, m \geq 1
$$

For each $\ell \geq 1$ we associate to such a family an asymptotic multiplier ideal $\mathcal{J}\left(\left\|\mathfrak{a}_{\ell}\right\|\right) \subseteq$ $\mathcal{O}_{X}$ which reflects the asymptotic behavior of all the ideals $\mathfrak{a}_{p \ell}$ for $p \gg 0$. Using the subadditivity theorem of [3], we prove

Theorem B. If $\mathcal{J}\left(\left\|\mathfrak{a}_{\ell}\right\|\right) \subseteq \mathfrak{b}$ for some index $\ell$ and some ideal $\mathfrak{b}$, then

$$
\mathfrak{a}_{m \ell} \subseteq \mathfrak{b}^{m}
$$

for every $m \geq 1$.

In the case of symbolic powers it is elementary to check that $\mathcal{J}\left(\left\|\mathfrak{q}^{(e)}\right\|\right) \subseteq \mathfrak{q}$, so Theorem A follows from the "abstract" Theorem B. As another application, we establish a result (Theorem 2.6) rendering effective and extending in certain directions a theorem of Izumi [9], [7] dealing with ideals arising from a valuation.

We have been guided by the viewpoint that the families $\left\{\mathfrak{a}_{k}\right\}$ share some of the behavior of the linear series $|k D|$ associated to multiples of a divisor $D$ on a projective variety, and that one can try to adapt geometric tools to the present setting. We hope that these and other ideas from higher dimensional complex geometry will find further algebraic applications in the future. Going in the other direction, Hochster and Huneke [6] have used the theory of tight closure to reprove and generalize the results of the present paper dealing with symbolic powers: in paticular they show that Theorem A holds for any regular local ring containing a field, and they remove the hypothesis that $\mathfrak{q}$ be radical (see $\S 3$ for further discussion). This illustrates once again the close but somewhat mysterious connections between tight closure methods and the more geometric outlook appearing here.

Our exposition is organized into two sections. In the first, we construct the multiplier ideals we use and establish their basic properties. The applications are given in $\S 2$.

\footnotetext{
${ }^{3}$ Lipman called them "adjoint ideals", but "multiplier ideal" has become standard in higher dimensional geometry. The name derives from their analytic construction, where they arise as sheaves of multipliers (see 肘).
} 
We are grateful to Mel Hochster and Craig Huneke for valuable discussions and encouragement, and to Jessica Sidman for some Macaulay scripts related to Example 2.3. We also wish to record our debt to the work of Irena Swanson and her collaborators, through which we learned of many of the questions discussed here.

\section{Graded Families And Multiplier IdeAls}

In this section we construct the multiplier ideals we require, and give their basic properties. Quick overviews of the general theory of multiplier ideals appear in [4] and [3], $§ 1$, and a survey of some of the applications in algebraic geometry is given in [2]. The forthcoming book 11] will contain a detailed exposition, which in the meantime can be found in the lecture notes [12]. In particular, [12] contains full proofs of all the facts about multiplier ideals quoted in the following paragraphs.

Let $X$ be a non-singular complex quasi-projective variety, and $\mathfrak{a} \subseteq \mathcal{O}_{X}$ a non-zero ideal sheaf on $X$. A $\log$ resolution of $\mathfrak{a}$ is a projective birational map $\mu: X^{\prime} \longrightarrow X$, with $X^{\prime}$ non-singular, such that $\mathfrak{a} \cdot \mathcal{O}_{X^{\prime}}=\mathcal{O}_{X^{\prime}}(-F)$ for an effective Cartier divisor $F$ on $X$ with the property that the sum of $F$ and the exceptional divisor of $\mu$ has simple normal crossing support. Such resolutions can be construced by resolving the singularities of the blow-up of $\mathfrak{a}$. We write $K_{X^{\prime} / X}=K_{X^{\prime}}-\mu^{*} K_{X}$ for the relative canonical divisor of $X^{\prime}$ over $X$.

Given a rational number $c>0$, the multiplier ideal associated to $c$ and $\mathfrak{a}$ is defined by fixing a log resolution as above, and setting

$$
\mathcal{J}(X, c \cdot \mathfrak{a})=\mathcal{J}(c \cdot \mathfrak{a})=\mu_{*} \mathcal{O}_{X^{\prime}}\left(K_{X^{\prime} / X}-[c F]\right) .
$$

Here $c F$ is viewed as an effective Q-divisor, and its integer part $[c F]$ is defined by taking the integral part of the coefficient of each of its components. The fact that $\mathcal{J}(c \cdot \mathfrak{a})$ is indeed an ideal follows from the observation that $\mathcal{J}(c \cdot \mathfrak{a}) \subseteq \mu_{*} \mathcal{O}_{X^{\prime}}\left(K_{X^{\prime} / X}\right)=\mathcal{O}_{X}$. An important point is that this definition is independent of the $\log$ resolution $\mu$.

It follows immediately from the definition that if $c \in \mathbf{N}$, then $\mathcal{J}(c \cdot \mathfrak{a})=\mathcal{J}\left(\mathfrak{a}^{c}\right)$. This being so, we sometimes prefer to use "exponential notation" $\mathcal{J}\left(\mathfrak{a}^{c}\right)$ for the multiplier ideal $\mathcal{J}(c \cdot \mathfrak{a})$ for an arbitrary rational number $c>0$. Note that we are not trying to attach any actual meaning to the expression $c \cdot \mathfrak{a}$ or $\mathfrak{a}^{c}$ when $c$ is non-integral. Nonetheless, the possibility of being able to work with rational coefficients is critical in applications.

As a variant, given ideals $\mathfrak{a}, \mathfrak{b} \subseteq \mathcal{O}_{X}$, and rational numbers $c, d>0$, we define $\mathcal{J}((c \cdot \mathfrak{a}) \cdot(d \cdot \mathfrak{b}))$ (or $\mathcal{J}\left(\mathfrak{a}^{c} \cdot \mathfrak{b}^{d}\right)$ in exponential notation) by taking a common log resolution $\mu: X^{\prime} \longrightarrow X$ of $\mathfrak{a}$ and $\mathfrak{b}$, with $\mathfrak{a} \cdot \mathcal{O}_{X^{\prime}}=\mathcal{O}_{X^{\prime}}\left(-F_{1}\right), \mathfrak{b} \cdot \mathcal{O}_{X^{\prime}}=\mathcal{O}_{X^{\prime}}\left(-F_{2}\right)$, and putting $\mathcal{J}\left(\mathfrak{a}^{c} \cdot \mathfrak{b}^{d}\right)=\mu_{*} \mathcal{O}_{X^{\prime}}\left(K_{X^{\prime} / X}-\left[c F_{1}+d F_{2}\right]\right)$. It is sometimes useful also to adopt the convention that if $\mathfrak{a}=(0)$, then $\mathcal{J}(c \cdot \mathfrak{a})=(0)$ for all $c>0$.

The most important local property of multiplier ideals is the Restriction Theorem, due in the algebro-geometric setting to Esnault and Viehweg. Specifically, let $Y \subseteq X$ be a smooth subvariety, and let $\mathfrak{a} \subseteq \mathcal{O}_{X}$ be an ideal sheaf whose zeroes do not contain $Y$. Then $\mathfrak{a}_{Y}=\mathfrak{a} \cdot \mathcal{O}_{Y}$ is an ideal sheaf on $Y$, and the result in question states that one has 
an inclusion:

$$
\mathcal{J}\left(Y, c \cdot \mathfrak{a}_{Y}\right) \subseteq \mathcal{J}(X, c \cdot \mathfrak{a}) \cdot \mathcal{O}_{Y}
$$

of ideal sheaves on $Y$. This is established by reducing to the case where $Y$ has codimension one, and applying vanishing theorems. The restriction theorem leads in turn to the Subaddivity Theorem of [3], which states (in exponential notation) that given ideals $\mathfrak{a}, \mathfrak{b} \subseteq$ $\mathcal{O}_{X}$ and rational numbers $c, d>0$, one has the inclusion:

$$
\mathcal{J}\left(\mathfrak{a}^{c} \cdot \mathfrak{b}^{d}\right) \subseteq \mathcal{J}\left(\mathfrak{a}^{c}\right) \cdot \mathcal{J}\left(\mathfrak{b}^{d}\right) .
$$

To prove this, one first of all applies the Künneth formula to check that

$$
\mathcal{J}\left(X \times X,\left(p_{1}^{-1} \mathfrak{a}\right)^{c} \cdot\left(p_{2}^{-1} \mathfrak{b}\right)^{d}\right)=p_{1}^{-1} \mathcal{J}\left(X, \mathfrak{a}^{c}\right) \cdot p_{2}^{-1} \mathcal{J}\left(X, \mathfrak{b}^{d}\right),
$$

where $p_{1}, p_{2}: X \times X \longrightarrow X$ are the projections, where we are somewhat abusively writing $f^{-1} \mathfrak{o} \subseteq \mathcal{O}_{V}$ for inverse image $\mathfrak{o} \cdot \mathcal{O}_{V}$ of an ideal $\mathfrak{o} \subseteq \mathcal{O}_{W}$ under a morphism $f: V \longrightarrow W$. Then one restricts to the diagonal. Note for later reference that in "additive notation" (2) implies

$$
\mathcal{J}(c m \cdot \mathfrak{a}) \subseteq \mathcal{J}(c \cdot \mathfrak{a})^{m}
$$

for every integer $m \geq 1$.

Some of the most interesting applications of multiplier ideals (for instance [14], [10]) depend on the fact that one can make asymptotic constructions. A natural algebraic setting for these is described in the following

Definition 1.1. A graded family or graded system of ideals $\mathfrak{a}_{\bullet}=\left\{\mathfrak{a}_{k}\right\}$ is a collection of ideal sheaves $\mathfrak{a}_{k} \subseteq \mathcal{O}_{X}(k \geq 1)$ satisfying

$$
\mathfrak{a}_{k} \cdot \mathfrak{a}_{\ell} \subseteq \mathfrak{a}_{k+\ell} \text { for all } k, \ell \geq 1
$$

To avoid unnecessary complications, we assume also that $\mathfrak{a}_{k} \neq(0)$ for $k \gg 0$.

Note that if we set $A_{0}=\mathcal{O}_{X}$, then condition (A) is equivalent to the statement that $\oplus_{k \geq 0} \mathfrak{a}_{k}$ is a graded $\mathcal{O}_{X}$-algebra. The asymptotic constructions that follow are particularly useful in case this algebra is not finitely generated (or at least not known to be so).

Example 1.2. (i). Let $(0) \neq \mathfrak{a} \subseteq \mathcal{O}_{X}$ be a fixed ideal, and take $\mathfrak{a}_{k}=\mathfrak{a}^{k}$ to be the $k^{\text {th }}$ power of $\mathfrak{a}$. Then the $\left\{\mathfrak{a}_{k}\right\}$ form a graded family. One should view this as a trivial example.

(ii). Let $D$ be a divisor on a projective variety $X$. When $H^{0}\left(X, \mathcal{O}_{X}(k D)\right) \neq 0$ let $\mathfrak{b}_{k}=\mathfrak{b}(|k D|)$ be the base-ideal of the complete linear series $|k D|$, and put $\mathfrak{b}_{k}=(0)$ otherwise. Then $\mathfrak{b}_{\bullet}=\left\{\mathfrak{b}_{k}\right\}$ forms a graded family of ideals.

(iii). Let $(0) \neq \mathfrak{q} \subseteq \mathcal{O}_{X}$ be a radical ideal. Then the symbolic powers $\left\{\mathfrak{q}^{(k)}\right\}$ form a graded family of ideals that we denote by $\mathfrak{q}^{(\bullet)}$.

(iv). Let $\nu: Y \longrightarrow X$ be a proper birational map, and let $D$ be a non-zero effective Cartier divisor on $Y$. Then we get a graded family of ideals $\mathfrak{o}_{\bullet}=\left\{\mathfrak{o}_{k}\right\}$ on $X$ by putting $\mathfrak{o}_{k}=\nu_{*} \mathcal{O}_{Y}(-k D)$. Note that this includes the symbolic powers $\mathfrak{q}^{(k)}$ in (iii) as a special case, as well as the graded family of ideals associated to an $\mathfrak{m}$-valuation on $X$ in the sense of [7]. 
(v). Let $p(t)=\sum_{i=1}^{\infty} \frac{1}{i !} t^{i} \in \mathbf{C}[[t]]$ be the power series of the function $e^{t}-1$, and given $f \in \mathbf{C}[x, y]$ define

$$
v(f)=\operatorname{ord}_{t} f(t, p(t)) .
$$

This is a valuation on $\mathbf{C}[x, y]$, and therefore the ideals

$$
\mathfrak{o}_{k}={ }_{\text {def }}\{f \in \mathbf{C}[x, y] \mid v(f) \geq k\}
$$

(which we may view as ideal sheaves on $X=\mathbf{C}^{2}$ ) form a graded family. Explicitly,

$$
\mathfrak{o}_{k}=\left(x^{k}, y-p_{k}(x)\right),
$$

where $p_{k}(t)=\sum_{i=1}^{k} \frac{1}{i !} t^{i}$ is the $k^{\text {th }}$ Taylor polynomial of $e^{t}-1$.

(vi). Assume that $X$ is affine (and as always non-singular), so that ideal sheaves are identified with ideals in the coordinate ring $\mathbf{C}[X]$ of $X$. Given any non-zero ideal $\mathfrak{a} \subseteq \mathbf{C}[X]$, set

$\mathfrak{a}^{\{k\}}=\{f \in \mathbf{C}[X] \mid D f \in \mathfrak{a} \forall$ differential operators $D$ on $X$ of order $<k\}$.

This determines a graded family $\mathfrak{a}^{\{\bullet\}}$ which also reduces to the symbolic powers $\left\{\mathfrak{q}^{(k)}\right\}$ when $\mathfrak{a}=\mathfrak{q}$ is radical.

(vii). Let $\mathfrak{a}_{\bullet}=\left\{\mathfrak{a}_{k}\right\}$ be a graded family, and $\mathfrak{b} \subseteq \mathcal{O}_{X}$ a fixed ideal. Then the colon ideals

$$
\mathfrak{r}_{k}=\left(\mathfrak{a}_{k}: \mathfrak{b}^{k}\right)={ }_{\text {def }}\left\{f \in \mathcal{O}_{X} \mid f \cdot \mathfrak{b}^{k} \in \mathfrak{a}_{k}\right\}
$$

form a graded family.

We now construct the asymptotic multiplier ideal associated to a graded family $\mathfrak{a}_{\bullet}$.

Lemma 1.3. Let $\mathfrak{a}_{\bullet}=\left\{\mathfrak{a}_{k}\right\}$ be a graded family of ideals, and fix $\ell \in \mathbf{N}$ plus a rational number $c>0$. Then for all positive integers $p, n \geq 1$ one has

$$
\mathcal{J}\left(\frac{c}{p} \cdot \mathfrak{a}_{p \ell}\right) \subseteq \mathcal{J}\left(\frac{c}{p n} \cdot \mathfrak{a}_{p n \ell}\right) .
$$

Proof. Let $\mu: X^{\prime} \longrightarrow X$ be a common $\log$ resolution of $\mathfrak{a}_{p \ell}$ and $\mathfrak{a}_{p n \ell}$, with

$$
\mathfrak{a}_{p \ell} \cdot \mathcal{O}_{X^{\prime}}=\mathcal{O}_{X^{\prime}}\left(-F_{p \ell}\right) \quad, \quad \mathfrak{a}_{p n \ell} \cdot \mathcal{O}_{X^{\prime}}=\mathcal{O}_{X^{\prime}}\left(-F_{p n \ell}\right) .
$$

Condition (四) implies that $\mathfrak{a}_{p \ell}^{n} \subseteq \mathfrak{a}_{p n \ell}$, and hence $-n F_{p \ell} \preccurlyeq-F_{p n \ell}$ (i.e. the difference $n F_{p \ell}-F_{p n \ell}$ is effective). Therefore

$$
K_{X^{\prime} / X}-\left[\frac{c}{p \ell} \cdot F_{p \ell}\right] \preccurlyeq K_{X^{\prime} / X}-\left[\frac{c}{p n \ell} \cdot F_{p n \ell}\right],
$$

and the statement follows.

We assert next that the collection of multiplier ideals

$$
\left\{\mathcal{J}\left(\frac{c}{p} \cdot \mathfrak{a}_{p \ell}\right)\right\}_{(p>0)}
$$

has a unique maximal element. In fact, the existence of one maximal element follows from the ascending chain condition on ideals. On the other hand, if $\mathcal{J}\left(\frac{c}{p} \cdot \mathfrak{a}_{p \ell}\right)$ and $\mathcal{J}\left(\frac{c}{q} \cdot \mathfrak{a}_{q \ell}\right)$ are both maximal, then thanks to the Lemma they each coincide with $\mathcal{J}\left(\frac{c}{p q} \cdot \mathfrak{a}_{p q \ell}\right)$. 
Definition 1.4. Given a graded family of ideals $\mathfrak{a}_{\bullet}=\left\{\mathfrak{a}_{k}\right\}$, the asymptotic multiplier ideal at level $\ell$ associated to $c>0$ and $\mathfrak{a}_{\bullet}$, written $\mathcal{J}\left(c \cdot\left\|\mathfrak{a}_{\ell}\right\|\right)$, is the maxmial element of the collection of ideals appearing in (5). In other words,

$$
\mathcal{J}\left(c \cdot\left\|\mathfrak{a}_{\ell}\right\|\right)=\mathcal{J}\left(\frac{c}{p} \cdot \mathfrak{a}_{p \ell}\right) \quad \text { for sufficiently divisible } p \gg 0
$$

Assuming as we are that $\mathfrak{a}_{k} \neq(0)$ for $k \gg 0$, one can show that there is an integer $p_{0}=p_{0}\left(\mathfrak{a}_{\bullet}, \ell\right)$ such that $\mathcal{J}\left(c \cdot\left\|\mathfrak{a}_{\ell}\right\|\right)=\mathcal{J}\left(\frac{c}{p} \cdot \mathfrak{a}_{p \ell}\right)$ for all $p \geq p_{0}$. We use this fact only to observe that one does not actually need the divisibility condition in (6).

Remark 1.5. Note that $\mathcal{J}\left(c \cdot\left\|\mathfrak{a}_{\ell}\right\|\right)$ depends not just on the particular ideal $\mathfrak{a}_{\ell}$, but on all the ideals $\mathfrak{a}_{p \ell}$ for $p \gg 0$. The double vertical lines should serve as a reminder of this point.

Example 1.6. (i). If $\mathfrak{a}_{k}=\mathfrak{a}^{k}$ is the trivial graded family consisting of powers of a fixed ideal $\mathfrak{a}$, then $\mathcal{J}\left(c \cdot\left\|\mathfrak{a}_{\ell}\right\|\right)=\mathcal{J}\left(c \cdot \mathfrak{a}^{\ell}\right)=\mathcal{J}(c \ell \cdot \mathfrak{a})$.

(ii). When $\mathfrak{b}_{k}=\mathfrak{b}(|k D|)$ is the family of base ideals associated to a big divisor $D$, then $\mathcal{J}\left(c \cdot\left\|\mathfrak{b}_{\ell}\right\|\right)=\mathcal{J}(c \cdot\|\ell D\|)$ is the asymptotic multiplier ideal constructed for instance in [10] and [11]. These ideals have played an important role in recent work on linear series.

(iii). Let $\mathfrak{q} \subseteq \mathcal{O}_{X}$ be a radical ideal. We denote the asymptotic multiplier ideal at level $\ell$ associated to the symbolic powers $\mathfrak{q}^{(\bullet)}=\left\{\mathfrak{q}^{(k)}\right\}$ by $\mathcal{J}\left(c \cdot\left\|\mathfrak{q}^{(\ell)}\right\|\right)$. Thus $\mathcal{J}\left(c \cdot\left\|\mathfrak{q}^{(\ell)}\right\|\right)=$ $\mathcal{J}\left(\frac{c}{p} \cdot \mathfrak{q}^{(p \ell)}\right)$ for $p \gg 0$.

(iv). Consider the ideals $\mathfrak{o}_{k} \subseteq \mathbf{C}[x, y]$ constructed in Example $1.2(\mathrm{v})$ associated to the valuation $v(f)=\operatorname{ord}_{t} f\left(t, e^{t}-1\right)$. Then $\mathcal{J}\left(\left\|\mathfrak{o}_{\ell}\right\|\right)=\mathbf{C}[x, y]$ for every $\ell$. This can be checked directly using the observation that each $\mathfrak{o}_{k}$ contains a polynomial whose divisor is a smooth curve. From a more sophisticated point of view, the triviality of the multiplier ideal in question is implied by Theorem B plus the fact that the colength of $\mathfrak{o}_{k}$ in $\mathbf{C}[x, y]$ grows linearly rather than quadratically in $k$.

For our purposes the essential properties of these multiplier ideals are given by

Proposition 1.7. Let $\mathfrak{a}_{\bullet}=\left\{\mathfrak{a}_{k}\right\}$ be a graded family of ideals on the smooth variety $X$, and fix $\ell \geq 1$. Then:

(i). $\mathfrak{a}_{\ell} \subseteq \mathcal{J}\left(\left\|\mathfrak{a}_{\ell}\right\|\right)$.

(ii). For every $m \geq 1$ one has the inclusion

$$
\mathcal{J}\left(\left\|\mathfrak{a}_{m \ell}\right\|\right) \subseteq \mathcal{J}\left(\left\|\mathfrak{a}_{\ell}\right\|\right)^{m}
$$

Proof. Since the relative canonical bundle $K_{X^{\prime} / X}$ is effective, it follows from the definition that $\mathfrak{a} \subseteq \mathcal{J}(\mathfrak{a})$ for any ideal $\mathfrak{a} \subseteq \mathcal{O}_{X}$. Then using Lemma 1.3 we find that

$$
\mathfrak{a}_{\ell} \subseteq \mathcal{J}\left(\mathfrak{a}_{\ell}\right) \subseteq \mathcal{J}\left(\frac{1}{p} \cdot \mathfrak{a}_{p \ell}\right)
$$


Taking $p \gg 0$, this gives (i). For (ii), fix $p \gg 0$ and use the subadditivity relation (3) to deduce:

$$
\begin{aligned}
\mathcal{J}\left(\left\|\mathfrak{a}_{m \ell}\right\|\right) & =\mathcal{J}\left(\frac{1}{p} \cdot \mathfrak{a}_{p m \ell}\right) \\
& =\mathcal{J}\left(\frac{m}{p m} \cdot \mathfrak{a}_{p m \ell}\right) \\
& \subseteq \mathcal{J}\left(\frac{1}{p m} \cdot \mathfrak{a}_{p m \ell}\right)^{m} \\
& =\mathcal{J}\left(\left\|\mathfrak{a}_{\ell}\right\|\right)^{m}
\end{aligned}
$$

as asserted.

Remark 1.8. Note that it need not be true in general that $\mathcal{J}\left(\mathfrak{a}_{m \ell}\right) \subseteq \mathcal{J}\left(\mathfrak{a}_{\ell}\right)^{m}$. This explains why it is crucial to pass to the asymptotic ideals.

\section{Applications}

Our concrete results follow from the following general statement — which appears as Theorem B in the Introduction - concerning the multiplicative behavior of graded families of ideals:

Theorem 2.1. Let $\mathfrak{a}_{\bullet}=\left\{\mathfrak{a}_{k}\right\}$ be a graded family of ideals on a smooth complex variety $X$, and suppose that $\mathfrak{b} \subseteq \mathcal{O}_{X}$ is an ideal such that $\mathcal{J}\left(\left\|\mathfrak{a}_{\ell}\right\|\right) \subseteq \mathfrak{b}$ for some index $\ell \in \mathbf{N}$. Then

$$
\mathfrak{a}_{m \ell} \subseteq \mathfrak{b}^{m}
$$

for every integer $m \geq 1$.

Proof. This is an immediate consequence of Proposition 1.7, which implies that

$$
\mathfrak{a}_{m \ell} \subseteq \mathcal{J}\left(\left\|\mathfrak{a}_{m \ell}\right\|\right) \subseteq \mathcal{J}\left(\left\|\mathfrak{a}_{\ell}\right\|\right)^{m}
$$

The first application is to symbolic powers:t

Theorem 2.2. Let $X$ be a smooth complex variety, and $Z \subseteq X$ a reduced subscheme all of whose irreducible components have codimension $\leq e$ in $X$. Put $\mathfrak{q}=\mathcal{I}_{Z}$, and fix an integer $\ell \geq e$. Then

$$
\mathfrak{q}^{(m \ell)} \subseteq\left(\mathfrak{q}^{(\ell+1-e)}\right)^{m}
$$

for every $m \geq 1$. In particular, taking $\ell=e$ one has

$$
\mathfrak{q}^{(m e)} \subseteq \mathfrak{q}^{m} \quad \text { for all } m \geq 1
$$

Proof. It suffices by Theorem 2.1 to show that

$$
\mathcal{J}\left(\left\|\mathfrak{q}^{(\ell)}\right\|\right) \subseteq \mathfrak{q}^{(\ell+1-e)}
$$

But membership in the ideal on the right is tested locally at a general point of each irreducible component of $Z$. So we can assume after shrinking $X$ that $Z$ is smooth and irreducible, of codimension $e$, and in this case $\left(^{*}\right)$ is clear. For then $\mathfrak{q}^{(k)}=\mathfrak{q}^{k}$ for all $k$, and

\footnotetext{
${ }^{4}$ See $\S 3$ for a more general statement.
} 
$\mathfrak{q}$ is resolved by taking $\mu: X^{\prime}=\mathrm{Bl}_{Z}(X) \longrightarrow X$ to be the blow-up of $X$ along $\mathfrak{q}$. Writing $E \subseteq X^{\prime}$ for the corresponding exceptional divisor, one has

$$
K_{X^{\prime} / X}=(e-1) E \text { and } \mathfrak{q}^{\ell} \cdot \mathcal{O}_{X^{\prime}}=\mathcal{O}_{X^{\prime}}(-\ell E) .
$$

Consequently

$$
\mathcal{J}\left(\left\|\mathfrak{q}^{(\ell)}\right\|\right)=\mu_{*} \mathcal{O}_{X^{\prime}}\left(K_{X^{\prime} / X}-\ell E\right)=\mu_{*} \mathcal{O}_{X^{\prime}}(-(\ell+1-e) E)=\mathfrak{q}^{l+1-e}
$$

as asserted.

Example 2.3. The first non-trivial case of Theorem 2.2 is the following. Let $T \subseteq \mathbf{P}^{2}$ be a finite set of points, viewed as a reduced algebraic subset of the plane, and let $I \subseteq$ $\mathbf{C}[X, Y, Z]$ be the homogeneous ideal of $T$. If $F \in \mathbf{C}[X, Y, Z]$ is a homogeneous polynomial having multiplicity $\geq 2 m$ at every point of $T$, then $F \in I^{m}$. (Apply Theorem 2.2 to the affine cone over $T$ in $\mathbf{C}^{3}$.) In spite of the very classical nature of this statement we do not know a direct elementary proof.

Remark 2.4. The statement of Theorem 2.2 can fail on singular varieties. For example Huneke points out that counter-examples arise already when $Z$ is a line on a quadric cone $X$ in $\mathbf{C}^{3}$. However Hochster and Huneke [6] give some statements valid also on singular ambient spaces.

Remark 2.5. Using familiar arguments, one can deduce from Theorem 2.2 that the corresponding statement holds for excellent regular local rings containing a field of characteristic zero. However Hochster and Huneke [6] have shown that in fact the analogue of (2.2) holds in any regular local ring containing a field. Therefore we do not dwell on the question finding the most general situation in which the arguments of the present paper apply.

We conclude with a result which renders effective and extends in certain directions a formulation due to Hübl and Swanson ([0], (1.4)) of a theorem of Izumi [9]:

Theorem 2.6. Let $\nu: Y \longrightarrow X$ be a proper birational map between smooth complex varieties. Let $E \subseteq Y$ be a prime divisor, set

$$
\ell=1+\operatorname{ord}_{E}\left(K_{Y / X}\right)
$$

and for $k \geq 1$ put $\mathfrak{o}_{k}=\nu_{*} \mathcal{O}_{Y}(-k E)$. Fix an irreducible subvariety $Z \subseteq X$ such that $Z \subseteq \nu(E)$ and denote by $\mathfrak{p}=\mathcal{I}_{Z}$ the ideal of $Z$. Then

$$
\mathfrak{o}_{\ell m} \subseteq \mathfrak{p}^{m} \quad \text { for all } m \geq 1
$$

Remark 2.7. The result discussed in [0] — which holds in considerably more general settings, but without the explicit determination of the coefficient $\ell$ of $m$ - deals with the situation in which $E$ maps to a point. It was in trying to understand this result that we were led to the statements about symbolic powers. 
Proof of Theorem 2.6. We can assume without loss of generality that $E$ is $\nu$-exceptional and that $Z=\nu(E)$, so that $\nu_{*} \mathcal{O}_{Y}(-E)=\mathfrak{p}$. Applying (2.1) to the graded family $\mathfrak{o}_{\bullet}=\left\{\mathfrak{o}_{k}\right\}$ (Example 1.2(iv)), it suffices to prove that $\mathcal{J}\left(\left\|\mathfrak{o}_{\ell}\right\|\right) \subseteq \mathfrak{p}$. We suppose to this end that we've fixed a large integer $p \gg 0$ such that the multiplier ideal $\mathcal{J}\left(\left\|\mathfrak{o}_{\ell}\right\|\right)=\mathcal{J}\left(\frac{1}{p} \cdot \mathfrak{o}_{p \ell}\right)$ in question is computed on a log resolution $\mu: X^{\prime} \longrightarrow X$ of $\mathfrak{o}_{p \ell}$ dominating $\nu: Y \longrightarrow X$. Then $E$ gives rise to a prime divisor on $E^{\prime}$ on $X^{\prime}$ - viz. the proper transform of $E$ with

$$
\operatorname{ord}_{E^{\prime}}\left(K_{X^{\prime} / X}\right)=\operatorname{ord}_{E}\left(K_{Y / X}\right)=\ell-1,
$$

and one has $\mathfrak{o}_{k}=\mu_{*} \mathcal{O}_{X^{\prime}}\left(-k E^{\prime}\right)$ for every $k \geq 1$.

Let $F$ be the effective Cartier divisor on $X^{\prime}$ defined in the usual way by writing $\mathfrak{o}_{p \ell} \cdot \mathcal{O}_{X^{\prime}}=\mathcal{O}_{X^{\prime}}(-F)$. Since $\mathfrak{o}_{p \ell}=\mu_{*} \mathcal{O}_{X^{\prime}}\left(-p \ell E^{\prime}\right)$, we see that $E^{\prime}$ appears with coefficient $\geq p \ell$ in $F$. Consequently

$$
\operatorname{ord}_{E^{\prime}}\left(K_{X^{\prime} / X}-\left[\frac{1}{p} F\right]\right) \leq(\ell-1)-\ell=-1
$$

and therefore

$$
\mathcal{J}\left(\left\|\mathfrak{o}_{\ell}\right\|\right)=\mu_{*} \mathcal{O}_{X^{\prime}}\left(K_{X^{\prime} / X}-\left[\frac{1}{p} F\right]\right) \subseteq \mu_{*} \mathcal{O}_{X^{\prime}}\left(-E^{\prime}\right)=\mathfrak{p},
$$

as required.

\section{Generalizations}

In the preprint [6], which appeared shortly after the first version of the present paper, Hochster and Huneke use the theory of tight closure to extend Theorem 2.2 to arbitrary regular local rings containing a field. They also observe that it is sufficient to assume that $\mathfrak{q} \subseteq \mathcal{O}_{X}$ is unmixed. In this section we indicate how one applies Theorem 2.1 to treat unmixed ideals.

We start by recalling the definition of symbolic powers in this more general setting. Assume for simplicity of exposition that the smooth complex variety $X$ is affine. Given an ideal $\mathfrak{q} \subseteq \mathbf{C}[X]$, fix a primary decomposition

$$
\mathfrak{q}=\mathfrak{q}_{1} \cap \ldots \cap \mathfrak{q}_{h}
$$

of $\mathfrak{q}$, and let $Y_{i}=\operatorname{Zeroes}\left(\sqrt{\mathfrak{q}_{i}}\right)$ be the subvarieties of $X$ corresponding to the associated primes $\mathfrak{p}_{i}=\sqrt{\mathfrak{q}_{i}}$ of $\mathfrak{q}$. Recall that $\mathfrak{q}$ is unmixed if none of the associated primes $\mathfrak{p}_{i}$ are embedded (or equivalently if there are no inclusions among the $Y_{i}$ ). Then the symbolic powers $\mathfrak{q}^{(k)} \subseteq \mathbf{C}[X]$ of $\mathfrak{q}$ are defined as follows. For each associated subvariety $Y_{i}$ of $\mathfrak{q}$, there is a natural map $\phi_{i}: \mathbf{C}[X] \longrightarrow \mathcal{O}_{Y_{i}} X$ from the coordinate ring of $X$ to the local ring of $X$ along $Y_{i}$. We then set

$$
\mathfrak{q}^{(k)}=\bigcap_{i=1}^{h} \phi_{i}^{-1}\left(\mathfrak{q}^{k} \cdot \mathcal{O}_{Y_{i}} X\right) .
$$

In other words, $f \in \mathfrak{q}^{(k)}$ if and only if there is an element $s \in \mathbf{C}[X]$, not lying in any of the associated primes $\mathfrak{p}_{i}$ of $\mathfrak{q}$, such that $f s \in \mathfrak{q}^{k}$. 
Theorem 2.2 then admits the following

Variant. Let $\mathfrak{q} \subseteq \mathbf{C}[X]$ be an unmixed ideal, and assume that every associated subvariety $Y_{i}$ of $\mathfrak{q}$ has codimension $\leq e$ in $X$. Then $\mathfrak{q}^{(m e)} \subseteq \mathfrak{q}^{m}$ for all natural numbers $m \geq 1$.

Sketch of Proof. The symbolic powers $\mathfrak{q}^{(\bullet)}=\left\{\mathfrak{q}^{(k)}\right\}$ again form a graded family of ideals, so Theorem 2.1 will apply as soon as we establish that $\mathcal{J}\left(\left\|\mathfrak{q}^{(e)}\right\|\right) \subseteq \mathfrak{q}$. Referring to the primary decomposition $(*)$, it is enough to show that

$$
\mathcal{J}\left(\left\|\mathfrak{q}^{(e)}\right\|\right) \subseteq \mathfrak{q}_{i} \text { for each } 1 \leq i \leq h .
$$

For a given index $i$, inclusion in $\mathfrak{q}_{i}$ is tested at a generic point of $Y_{i}$. So having fixed $i$ we are free to replace $X$ by any open subset meeting $Y_{i}$. Therefore, by definition of the symbolic powers, we may assume after localizing that $\mathfrak{q}^{(k)}=\mathfrak{q}^{k}$. But in this case $\mathcal{J}\left(\left\|\mathfrak{q}^{(e)}\right\|\right)=\mathcal{J}\left(\mathfrak{q}^{e}\right)$, and $\mathcal{J}\left(\mathfrak{q}^{e}\right) \subseteq \mathfrak{q} \subseteq \mathfrak{q}_{i}$ thanks to a variant of a theorem of Skoda (cf. [12]).

Remark 3.1. While there are certain similarities of spirit between the arguments appearing here and those of Hochster and Huneke [6] - e.g. both involve asymptotic constructions, and reduce to the situation in which $\mathfrak{q}^{(k)}=\mathfrak{q}^{k}$ - the precise connections between the two points of view remain quite mysterious. In the hopes of understanding these connections more clearly, it is interesting to observe that the properties of multiplier ideals used here can be "axiomatized" as follows. Given a graded family $\mathfrak{q}_{\bullet}=\left\{\mathfrak{q}_{k}\right\}$ what is required for the application to symbolic powers is the existence of ideals $\mathcal{H}\left(\left\|\mathfrak{q}_{m}\right\|\right) \subseteq \mathcal{O}_{X}$ satisfying the following properties:

(i). $\mathcal{H}\left(\left\|\mathfrak{q}_{m}\right\|\right)$ is a sheaf on $X$, i.e. it commutes with localization, and when $\mathfrak{q}_{k}=\mathfrak{a}^{k}$ is the trivial family consisting of powers of a fixed ideal $\mathfrak{a}$, then Skoda's theorem

$$
\mathcal{H}\left(\left\|\mathfrak{a}^{n}\right\|\right) \subseteq \mathfrak{a}
$$

holds:?

(ii). $\mathfrak{q}_{m} \subseteq \mathcal{H}\left(\left\|\mathfrak{q}_{m}\right\|\right)$ for all $m$;

(iii). One has the subadditivity relation:

$$
\mathcal{H}\left(\left\|\mathfrak{q}_{\ell m}\right\|\right) \subseteq \mathcal{H}\left(\left\|\mathfrak{q}_{m}\right\|\right)^{\ell}
$$

In our setting, the required ideals are of course given by the asymptotic multiplier ideals $\mathcal{J}\left(\left\|\mathfrak{q}_{m}\right\|\right)$. However the existence of such ideals $\mathcal{H}$ is a purely algebraic question, and it would be very interesting to give a construction e.g. using ideas from tight closure. The hope here is that such a construction might serve as a Rosetta stone to help in deciphering the connections between the methods of the present note and the theory of tight closure.

\footnotetext{
${ }^{5}$ One also could ask for more precise statements involving the codimensions of associated primes of $\mathfrak{a}$.
} 


\section{REFERENCES}

[1] J.-P. Demailly, $L^{2}$ vanishing theorems for positive line bundles and adjunction theory, Lecture Notes of the CIME Session "Transcendental methods in Algebraic Geometry", Cetraro, Italy, July 1994, Ed. F. Catanese, C. Ciliberto, Lecture Notes in Math., Vol. 1646, 1-97.

[2] J.-P. Demailly, Méthodes $L^{2}$ et résultats effectifs en géométrie algébrique, Séminaire Bourbaki, exposé $\mathrm{n}^{\circ} 852$, novembre 1998, 38pp.

[3] J.-P. Demailly, L. Ein and R. Lazarsfeld, A subadditivity property of multiplier ideals, to appear in the special issue of the Mich. Math. J. in honor of W. Fulton.

[4] L. Ein, Multiplier ideals, vanishing theorems and applications, in Algebraic Geometry - Santa Cruz 1995, Proc. Symp. Pure Math. 62 (1997), pp. $203-219$.

[5] D. Eisenbud, Commutative Algebra, with a view towards algebraic geometry, Graduate Texts in Math. 150, Springer-Verlag, New York, 1995.

[6] M. Hochster and C. Huneke, Comparison of symbolic and ordinary powers of ideals, preprint.

[7] R. Hübl and I. Swanson, Discrete valuations cenetered on local domains, preprint (1998).

[8] C. Huneke, Uniform bounds in Noetherian rings, Inv. Math. 107 (1992), pp. 202 - 223.

[9] S. Izumi, A measure of integrity for local analytic algebras, Publ. RIMS Kyoto Univ. 21 (1985), pp. 719-735.

[10] Y. Kawamata, Deformations of canonical singularities, Journal of the Amer. Math. Soc. 12 (1999), 519-527.

[11] R. Lazarsfeld, Positivity in Algebraic Geometry, book in preparation.

[12] R. Lazarsfeld, Multiplier ideals for algebraic geometers, informal lecture notes available at http: //www.math. Isa.umich.edu/ rlaz

[13] J. Lipman, Adjoints of ideals in regular local rings, Math. Res. Lett. 1 (1994), pp. 739 - 755.

[14] Y.-T. Siu, Invariance of plurigenera, Invent. Math. 134 (1998), 661-673.

[15] I. Swanson, Linear equivalence of topologies, preprint (1997).

Department of Mathematics, University of Illinois at Chicago

851 South Morgan Street (M/C 249), Chicago, IL 60607-7045, USA

E-mail address: ein@math.uic.edu

Department of Mathematics, University of Michigan, Ann Arbor, Mi 48109, USA

E-mail address: rlaz@math.lsa.umich.edu

Department of Mathematics, University of Michigan, Ann Arbor, Mi 48109, USA

E-mail address: kesmith@math.1sa.umich.edu 